\title{
Potential role of TRAns Cervical Endosonography (TRACE) in brachytherapy of cervical cancer: proof of concept
}

\author{
Primoz Petric, MD, MSl'.2, Christian Kirisits, PhD ${ }^{3}$ \\ 'National Center for Cancer Care and Research, Hamad Medical Corporation, Doha, Qatar, ${ }^{2}$ Department of Brachytherapy, \\ Sector of Radiotherapy, Institute of Oncology Ljubljana, Ljubljana, Slovenia, ${ }^{3}$ Department of Radiotherapy, Comprehensive Cancer Center \\ Medical University of Vienna, Vienna, Austria
}

\begin{abstract}
Purpose: Magnetic resonance imaging (MRI) is the gold standard for image guided adaptive brachytherapy (BT) of cervical cancer. Ultrasound is an attractive alternative with reasonable costs and high soft tissue depiction quality. This technical note aims to demonstrate the proof of principle for use of TRAns Cervical Endosonography with rotating transducer in the context of brachytherapy (TRACE BT).

Material and methods: TRACE BT presentation is based on a single stage IIB cervical cancer patient. Prior to second BT implant, rotating US transducer $(6.9 \mathrm{~mm}$ diameter) was inserted in cervical canal and axial images obtained at $10 \mathrm{MHz}$, focal range of $30 \mathrm{~mm}$, and axial resolution of $0.4 \mathrm{~mm}$. Size and topography of hypo-echoic areas were assessed and optimal positions of interstitial needles were determined. Finally, intracavitary applicator was placed and needles inserted through vaginal ring-template according to TRACE pre-plan. MRI-based high risk clinical target volume $\left(\mathrm{CTV}_{\mathrm{HR}}\right)$ dimensions were compared with hypoechoic areas on TRACE. Topography of parametrial needles on post-insertion MRI was compared with TRACE pre-plan.

Results: Insertion of rotating mechanism into cervico-uterine cavity was safe, feasible and fast. The $360^{\circ}$ imaging in axial plane enabled real-time assessment of cervix, uterus, and adjacent parametria. Qualitative comparison of TRACE with post-insertion MRI revealed favorable agreement of findings. In-plane size of $\mathrm{CTV}_{\mathrm{HR}}$ on MRI was comparable to hypoechoic areas on TRACE. Needle positions on post-insertion MRI corresponded to TRACE-based pre-plan. Main limitation of TRACE was gradual deterioration of image quality due to coupling gel removal.

Conclusions: Present proof of concept demonstrates potential role of TRACE-BT for cervical cancer as an attractive high-tech approach with reasonable costs. Prior to investigation of its clinical role, further development of TRACE methodology is needed. This includes reliable transducer-tissue coupling, applicator reconstruction, imaging range, limitations in extensive tumors, US-based contouring concepts, registration with other imaging methods, organ dose-assessment, real-time dosimetry, etc.
\end{abstract}

J Contemp Brachytherapy 2016; 8, 3: 217-222 DOI: $10.5114 / \mathrm{jcb} .2016 .60502$

Key words: brachytherapy, cervical cancer, image guidance, ultrasound.

\section{Purpose}

Image guided adaptive brachytherapy (IGABT) improves dosimetric and clinical results of definitive chemoradiation in inoperable cervical cancer [1,2]. Utilization of novel intracavitary / interstitial applicators, personalized adjustment of application technique, and high quality sectional imaging are pre-requisites for dose optimization $[3,4,5,6,7]$. Currently, magnetic resonance imaging (MRI) is the recommended gold-standard modality for IGABT because of its high soft-tissue depiction quality $[8,9,10]$. However, high cost and complexity of MRI are the main impediments for its widespread use, especially in developing countries where cervical cancer is endemic [11]. Consequently, development of alternative imaging solutions in the context of IGABT is fundamental to the efforts of expanding access to the state of the art of cancer treatments and improving women's health on global scale. In addition, real-time imaging for insertion guidance and optimization of IGABT workflow is of interest for all centers in general.

Computed tomography (CT) has been studied as a relatively inexpensive alternative to MRI, and recommendations for CT based target volume contouring were 
published recently [12]. However, CT is characterized by inherent limitations when compared with MRI, especially for the delineation of the target volume. Even the often cited notion that $\mathrm{CT}$ is adequate for the organs at risk (OAR) delineation can be considered as an oversimplification. This notion is based on a single small published series [13], and is not generally supported by our clinical experience. In addition, the results of inter-observer studies indicate inferior results of CT-based organ contouring $[14,15,16]$. Hybrid approach with MRI at first $\mathrm{BT}$, followed by CT at subsequent fraction (s) has been suggested as a solution to reduce costs of IGABT while maintaining treatment accuracy [17]. While reliable and reproducible for selected patients, this method fails to eliminate MRI from the treatment planning process.

Ultrasound (US) has several advantages over MRI and CT. It is widely available, enables real-time image guidance during the procedure, and offers good depiction of soft tissues. Clinicopathological studies demonstrate comparable accuracy of ultrasound and MRI for assessment of primary tumor in early disease [18] as well as residual tumor in locally advanced cases treated with preoperative chemoradiation $[19,20]$. Recent comparison of trans-rectal ultrasound (TRUS) and MRI in inoperable cases treated with definitive chemoradiation demonstrated high correlation between the two methods, indicating potential of TRUS for target definition in IGABT [21]. Ultrasound has long tradition in BT with numerous publications on its use in the assessment of the intrauterine tandem position and real-time interstitial needle placement [22,23,24,25,26,27,28]. Recently, the use of US for treatment planning has been reported but the proposed approach did not eliminate MRI from the workflow $[29,30,31,32,33]$. In summary, the literature review demonstrates that the major potential of US for IGABT of cervical cancer extends beyond its conventional applications and may play a role in treatment planning. However, further research and development is required before it can be systematically implemented as a more widely available alternative to the current gold-standard [34].

In the present technical note, we propose an innovative and promising concept of real-time US imaging for cervical cancer BT - TRAns Cervical Endosonography (TRACE BT). Trans-cervical endosonography is a recognized medical procedure for diagnostic assessment and therapeutic interventions in the cervix and uterus $[35,36]$. In IGABT of anal cancer, the use of endo-anal rotating US probe has been reported as safe and feasible technique that enables real-time guidance of insertion and offers better information for dose planning when compared with CT $[37,38,39]$. To our knowledge, the potential of trans-cervical US with rotating transducer has never been explored in gynecological BT. At the author's institution, we have experience with using TRACE prior to BT procedure in selected patients with locally advanced tumors to aid tumor visualization and implant technique. This technical note summarizes our initial experience, demonstrated on a single patient case report and should be regarded as a proof of concept that warrants further research.

\section{Material and methods}

The theoretical design of TRACE BT system is presented on an example of endosonographic imaging findings in a patient with stage IIB cervical cancer with bilateral parametrial extension. The patient underwent whole pelvis external beam irradiation (50.4 Gy in $1.8 \mathrm{~Gy}$ daily fractions) with concomitant chemotherapy (40 mg/ $\mathrm{m}^{2}$ of cisplatin weekly), followed by two fractions of MRI pulsed dose rate IGABT, delivered in 25 hourly pulses. Details of our BT schedule, planning aims, and dose-optimization strategy are presented in [5]. Directly prior to the second BT implant, TRACE was performed under sterile conditions with the patient in lithotomy position and under spinal anesthesia.

Basic US-scanner settings were prepared in advance. The probe, transducer cable, and scanner keyboard were covered with sterile foil before patient transport to procedure room. Following the usual dilatation of cervical canal, a rotating US transducer with $6.9 \mathrm{~mm}$ diameter and sector angle of $360^{\circ}$ (BK Medical, type 1850 with 6005 transducer head, B\&K Medical, Herlev, Denmark) was covered with sterile coupling gel and manually inserted into the cervical canal. At a frequency of $10 \mathrm{MHz}$, US images were obtained with a focal range of $30 \mathrm{~mm}$ and axial resolution of $0.4 \mathrm{~mm}$ (measured at $25 \mathrm{~mm}$ ), using the Flex Focus 400 scanner (BK Medical). Images were obtained in axial plane at the levels of the cervix and uterus that were considered representative by the operator. Size and topography of the hypo-echoic areas, corresponding to the residual pathological tissues in the parametria were assessed. Based on this assessment, the target volume regions where interstitial needles would improve dose conformity were determined. After completion of the TRACE examination, the rotating probe was removed and intracavitary tandem and ring placed in the uterus and vagina. Finally, the interstitial needles were inserted through the vaginal ring template $[3,4]$ according to the TRACE preplan. Vaginal packing was performed, followed by MRIbased IGABT treatment planning. TRACE images were not used for dose planning or co-registration with MRI. They were qualitatively compared with post-insertion para-transverse T2 weighted fast spin echo MRI. Quantitative assessment was limited to comparison of maximal latero-lateral and antero-posterior dimensions of the MRIbased high risk clinical target volume $\left(\mathrm{CTV}_{\mathrm{HR}}\right)$ with corresponding dimensions of hypoechoic areas on TRACE. The positions of parametrial needles on post-insertion MRI were compared with the TRACE pre-plan.

\section{Results}

Ultrasound scanner setup and probe preparation prior to the procedure enabled fast and feasible TRACE imaging, resulting in 10 minutes prolongation of the BT procedure. Small diameter of the transducer $(6.9 \mathrm{~mm})$, which is comparable to the intrauterine applicator diameter $(6 \mathrm{~mm})$, resulted in feasible and safe insertion of the rotating mechanism into the cervico-uterine cavity. Mechanical rotating probe enabled $360^{\circ}$ axial imaging with refreshment rate that enabled clinically useful real-time 
assessment of pathoanatomy. Main relative limitation of the approach was gradual deterioration of image quality due to removal of the coupling gel, which was challenging in parts with tumor-related irregularities of the cervical canal.

The interval between TRACE and post-insertion MRI was 57 minutes. Results of the qualitative comparison of findings between both modalities were favorable (Figure 1). TRACE imaging enabled accurate depiction of the cervix, uterus, and adjacent parametrial tissues. Direct qualitative comparison of intraoperative TRACE with post-insertion MRI revealed favorable spatial agreement between sonographically suspicious bilateral hypoechoic areas and the grey zones, visible on the MRI (Figure 1). In-plane size of the $\mathrm{CTV}_{\mathrm{HR}}$ on MRI was comparable to hypoechoic areas on TRACE with maximum latero-lateral and anteroposterior dimensions differing for up to 2 and $3 \mathrm{~mm}$, respectively. While nearby OAR (rectum,
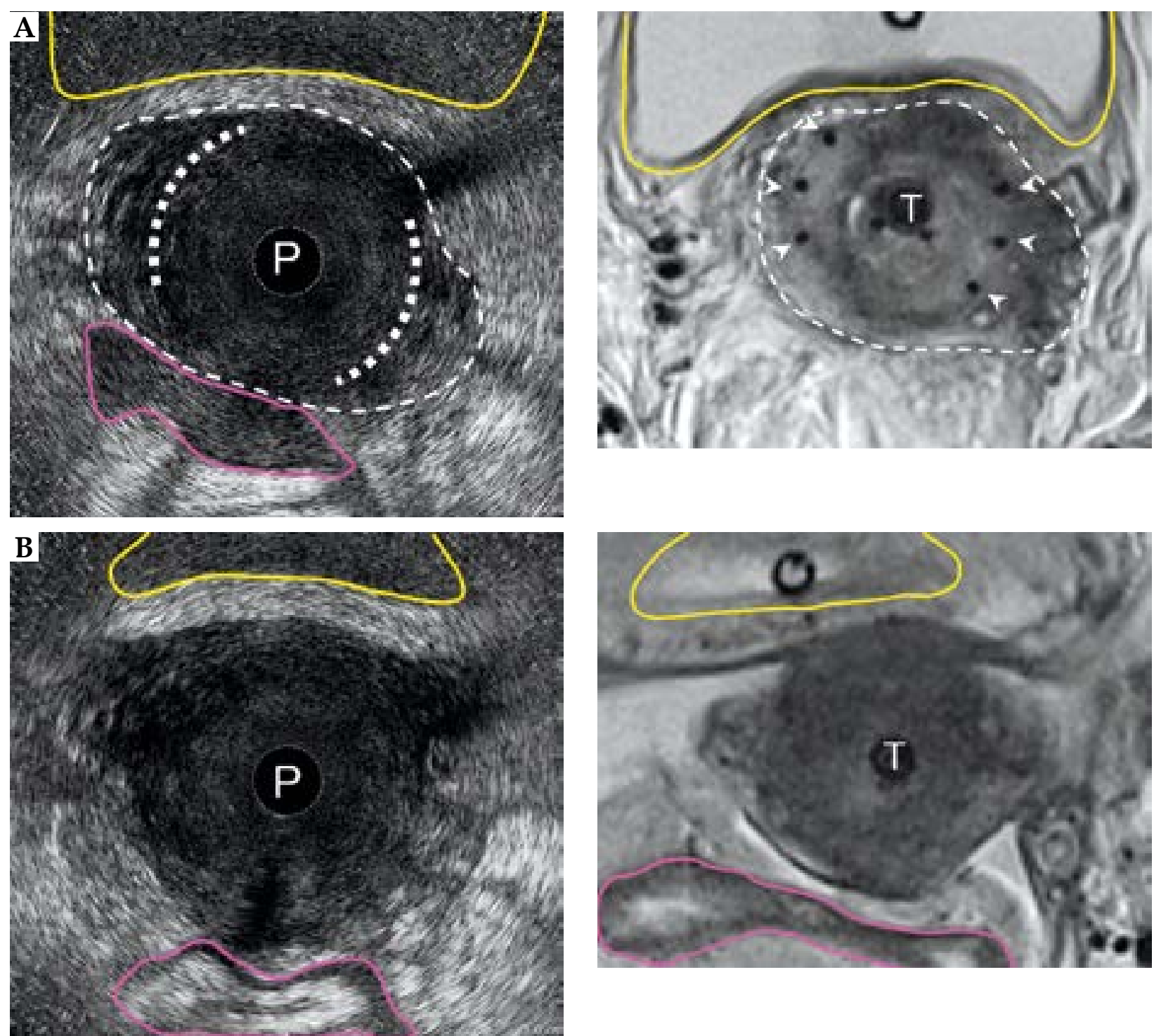

Fig. 1. Free-hand TRAns Cervical Endosonography (TRACE - left) compared to the T2 weighted fast spin echo magnetic resonance imaging (MRI) with the applicator in place (right) in the same patient at two different levels (A and $\mathbf{B})$. The MRI slices are cropped and oriented to show the area of interest, which corresponds to the TRACE field of view: due to the manual acquisition of the TRACE images, slight rotational post-processing in axial plane was required to enable comparisons. TRACE and MRI were obtained with 57 minutes interval. (A) Representative slice at the level of the target volume. The cervix and suspicious extra-cervical hypoechoic areas on TRACE correspond in size and topography to the high risk clinical target volume depicted on the MRI (thin white dotted lines). Thick dotted lines on the TRACE image (left) represent areas where interstitial needles would be beneficial. Actual needle positions on MRI (right) are depicted by arrowheads and correspond to the pre-planned areas on TRACE. Note the difference in OAR topography between TRACE and MRI. Sigmoid colon loop (magenta) that is visible on TRACE, is not present in the same position on MRI. Bladder filling and topography of posterior bladder wall also differ between modalities. (B) Representative slice through the uterine corpus (above the target volume). P - rotating endosonographic probe, inserted in the cervical canal, $\mathrm{T}$ - tandem applicator 
sigmoid, colon, and bladder) were well visible by both modalities, their location in relation to the target volume differed substantially (Figure 1). All needles were inserted inside the $\mathrm{CTV}_{\mathrm{HR}}$ in the regions corresponding to TRACE-based pre-plan (Figure 1).

\section{Discussion}

To our knowledge, this technical note represents the first documented application of trans-cervical rotating probe endosonography in gynecological BT. Experience presented here is limited to a single-case. It should be regarded as a proof of concept and not as a feasibility and safety study. Our results indicate that TRACE may play an important role in the future of cervical cancer IGABT and merits further systematic investigation.

Trans-abdominal, trans-rectal, and trans-vaginal US play an established role in preventing uterine perforations $[22,25,27,28,40]$ and real-time guidance of the insertion of interstitial needles $[23,24,26]$. Stock et al. demonstrated usefulness of trans-rectal ultrasound for real-time image guidance during interstitial implantation in 12 patients with gynecological malignancies [24]. In a study by Weitmann et al., ultrasound guidance of needle insertion was applied in 23 patients with vaginal recurrences of cervical and endometrial carcinoma [26]. The authors concluded that the procedure was feasible and safe, resulting in encouraging clinical results. More recently, Sharma et al. reported on $40 \mathrm{BT}$ procedures in 25 patients with locally advanced cervical cancer, using trans-rectal ultrasound for image guided placement of interstitial needles. Apart from transient hematuria in one patient, there was no acute toxicity and no organ perforation was identified on the post-implant CT [23]. Other authors have reported on similar results, demonstrating safety and feasibility of ultrasound for real-time guidance of intracavitary and/or interstitial applicator insertion. Van Dyk et al. found high level of inter-operator reliability when using trans-abdominal ultrasound to assess the uterus and cervix with the applicator in place [29]. The same group reported on promising results of combined use of MRI and US in cervical cancer BT planning. In their experience, intraoperative trans-abdominal US enables accurate target volume assessment and treatment planning, and translates into favorable clinical outcome $[29,30,31,32,33]$. While promising, the proposed approach does not eliminate the MRI from the workflow and, therefore, only partially addresses the problem of global availability of high-tech IGABT.

Potential advantages of TRACE over conventional US approaches, hypothetical methods of TRACE utilization, and current limitations of TRACE system are discussed below.

\section{Acoustic shadow and frame of reference}

US-guided insertion techniques that are reported in the literature and summarized above $[21,22,23,24,25,26$, $27,28,29,30,31,32,33,34]$ are based on placement of the US probes outside the target volume. These techniques are characterized by inherent limitations, which include different frames of reference of the transducer and the BT applicator as well as distortion of image quality by inserted applicators. The main difference between these methods and our concept is that the rotating TRACE probe and BT tandem are located inside the cervical canal and thus related to the same frame of reference during endosonography and treatment, respectively. Consequently, the problem of tandem-induced acoustic shadow during US imaging is eliminated. In addition, movement of the probe (or tandem) results in the corresponding movement of the target volume, and vice versa. This setup enables the "tandem-eye-view" of the target volume by the TRACE probe, which is independent of patient's setup. In present report, TRACE imaging and applicator placement were performed in lithotomy position, while MRI for treatment planning was obtained in supine position. In spite of this difference in setup, the relative topography between the target volume and the tandem on MRI corresponded to topography between the target volume and the probe on TRACE (Figure 1). However, this paradigm is only valid for the target volume and cannot be extrapolated to the OAR. Sectional imaging with the patient in treatment position, followed by co-registration [17] with TRACE imaging is needed for adequate assessment of spatial inter-relations between the OAR and the applicator.

\section{Intraoperative pre-planning of implant geometry}

Keeping the limitations of a single-patient report in mind, our results, experience, and mere intuition indicate that TRACE deserves further attention as promising tool for intraoperative pre-planning of implant geometry. We have used the information obtained from TRACE to plan the optimal position of interstitial needles, resulting in excellent topography of the final implant (Figure 1). Recently, MRI-based pre-planning has been shown to achieve excellent implant geometry and dosimetric results but implementation of these sophisticated methods remained limited to a few specialized centers [5,41]. Adaptations of TRACE system to the specific needs of gynecological BT, followed by its validation in clinical studies could result in a widely available and cost-effective alternative to MRI-based pre-planning in near future.

\section{Real time insertion guidance and treatment planning}

There is a longstanding experience with endosonography in the field of uro-oncology, including prostate BT and systems for trans-rectal and trans-urethral US-guided interventional diagnostic procedures. Endosonography-guided needle insertion has been employed in BT of anal cancer with excellent local control rates and minimal treatment-related morbidity $[37,38]$. In this tumor site, the use of endosonic rotating probes has been proven feasible and efficient in optimizing the implant procedure and treatment planning [39]. Trans-cervical endosonography has been used for diagnostic exploration of the cervix and uterus [35], and image guided radiofrequency ablation of uterine fibroids [36]. Existing technologies, developed in the above listed fields, include adjustable arms and steppers for ultrasound probes, template-based su- 
perimposition of needle path on the real-time images, and real-time dosimetry during BT implant. Our theoretical design of real-time TRACE BT system for target volume delineation and insertion guidance is currently under development. It is based on rigid connection between vaginal template for needle insertion and stepper-mounted rotating endosonic transducer. Due to the location of the probe inside the target volume, the artifacts during real-time TRACE-guided needle insertion are expected to be less pronounced when compared with conventional US setups (trans-abdominal, trans-rectal, and trans-vaginal). Assuming adequate vaginal packing for applicator immobilization, the relative position between the target and the applicator is not expected to change by altering the patient's position from lithotomy to supine. However, as discussed above, registration of TRACE images containing target volume with post-implant sectional imaging (i.e. CT) would be mandatory for delineation of the OAR and treatment planning, in order to account for positional changes of the OAR.

\section{Limitations}

In our experience, the main limitations of the presented prototype TRACE system were related to positional uncertainties due to free-hand probe handling and deterioration of image quality due to suboptimal coupling between the probe and the tissue at certain levels of the target volume (Figure 1). The probe that was employed in our report is not a part of a dedicated gynecological BT system. Therefore, several preconditions should be met prior to eventual clinical studies on TRACE BT for cervical cancer IGABT treatment planning. Technical adaptations of TRACE system, improved coupling, definition of the US-based target volume concepts, and their benchmarking against current gold-standard (MRI) are some of the issues, which need attention before clinical studies of TRACE-guided BT can be considered. It needs to be admitted that this technical note is based on our limited experience with a single patient case report, which has its major inherent limitations. Conclusions regarding feasibility, safety, and clinical utility of the procedure will require further studies, which we are planning as the next step. In spite of the above listed limitations, TRACE deserves further investigation as a promising and cost effective potential tool for cervical cancer IGABT.

\section{Conclusions}

This technical note is a proof of concept, demonstrating the potential role of TRACE in pre-planning, real-time guidance, and treatment planning of cervical cancer IGABT. Main limitations of currently available technology were identified and limitations of single patient based report acknowledged. Our favorable initial experience warrants further multidisciplinary research and development of a dedicated TRACE system for gynecological IGABT. The areas of investigation include effective transducer-tissue coupling, applicator reconstruction, imaging range, US contouring concepts and their validation, OAR dose assessment, registration with other imaging methods, and real-time dosimetry. These basic developments should be conducted before any clinical studies on feasibility, safety, and effectiveness of TRACE BT can be considered. Hypothetically, TRACE BT could be an interesting addition to complement existing imaging technologies in the future (MRI, CT, trans-abdominal US, trans-rectal US), to improve the image quality in the center of the target volume. When combined with $\mathrm{CT}$, TRACE BT could be a cost-effective alternative to the gold-standard MRI-based IGABT once the pre-conditions listed above are met.

\section{Acknowledgments}

The authors are grateful to Mr. Robert Hudej, PhD and Mr. Omar Hanuna from the Institute of Oncology Ljubljana, Department of Brachytherapy for their help and support in this work.

\section{Disclosure}

Authors report no conflict of interest.

\section{References}

1. Pötter R, Georg P, Dimopoulos JCA et al. Clinical outcome of protocol based image (MRI) guided adaptive brachytherapy combined with 3D conformal radiotherapy with or without chemotherapy in patients with locally advanced cervical cancer. Radiother Oncol 2011; 100: 116-123.

2. Lindegaard JC, Tanderup K, Nielsen SK et al. MRI-Guided 3D Optimization Significantly Improves DVH Parameters of Pulsed-Dose-Rate Brachytherapy in Locally Advanced Cervical Cancer. Int J Radiat Oncol Biol Phys 2008; 71: 756-764.

3. Kirisits C, Lang S, Dimopoulos J et al. The Vienna applicator for combined intracavitary and interstitial brachytherapy of cervical cancer: design, application, treatment planning, and dosimetric results. Int J Radiat Oncol Biol Phys 2006; 65: 624630.

4. Dimopoulos JCA, Kirisits C, Petric P et al. The Vienna applicator for combined intracavitary and interstitial brachytherapy of cervical cancer: clinical feasibility and preliminary results. Int J Radiat Oncol Biol Phys 2006; 66: 83-90.

5. Petric P, Hudej R, Hanuna O et al. MRI-assisted cervix cancer brachytherapy pre-planning, based on application in paracervical anaesthesia: final report. Radiol Oncol 2014; 48: 293300.

6. Lindegaard JC, Madsen ML, Traberg A et al. Individualized 3D printed vaginal template for MRI guided brachytherapy in locally advanced cervical cancer. Radiother Oncol 2016; 118: 173-175.

7. Nomden CN, de Leeuw AAC, Moerland MA et al. Clinical use of the Utrecht applicator for combined intracavitary/ interstitial brachytherapy treatment in locally advanced cervical cancer. Int J Radiat Oncol Biol Phys 2012; 82: 1424-1430.

8. Dimopoulos JC, Petrow P, Tanderup K et al. Recommendations from Gynaecological (GYN) GEC-ESTRO Working Group (IV): Basic principles and parameters for MRI imaging within the frame of image based adaptive cervix cancer brachytherapy. Radiother Oncol 2012; 103: 113-122.

9. Haie-Meder C, Pötter R, Van Limbergen E et al. Recommendations from Gynaecological (GYN) GEC-ESTRO Working Group (I): concepts and terms in 3D image based 3D treatment planning in cervix cancer brachytherapy with emphasis on MRI assessment of GTV and CTV. Radiother Oncol 2005; 74: $235-245$. 
10. Pötter R, Haie-Mader C, Van Limbergen E et al. Recommendations from gynaecological (GYN) GEC-ESTRO Working Group: (II): concepts and terms of 3D imaging, radiation physics, radiobiology, and 3D dose volume parameters. Radiother Oncol 2006; 78: 67-77.

11. Globocan 2013. Cervical Cancer Estimated Incidence, Mortality and Prevalence Worldwide in 2012. Available at: http:// globocan.iarc.fr/Pages/fact_sheets_cancer.aspx

12. Viswanathan AN, Erickson B, Gaffney DK et al. Comparison and consensus guidelines for delineation of clinical target volume for CT- and MR-based brachytherapy in locally advanced cervical cancer. Int J Radiat Oncol Biol Phys 2014; 90: 320-328.

13. Viswanathan AN, Dimopoulos JCA, Kirisits C et al. CT versus MRI-based contouring in cervical cancer brachytherapy: results of a prospective trial and preliminary guidelines for standardized Contours. Int J Radiat Oncol Biol Phys 2007; 68: 491-498.

14. Petric P, Hellebust TP, Tanderup K et al. Uncertainties of organs at risk delineation in MRI guided adaptive cervix cancer brachytherapy: A multi-institutional study. Eur J Cancer 2013; 49: S726.

15. Duane F, Langan B, Gillham C et al. Impact of delineation uncertainties on dose to organs at risk in CT-guided intracavitary brachytherapy. Brachytherapy 2014; 13: 210-218.

16. Saarnak AE, Boersma M, van Bunnigen BNFM et al. Interobserver variation in delineation of bladder and rectum contours for brachytherapy of cervical cancer. Radiother Oncol 2000; 56: 37-42.

17. Nesvacil N, Pötter R, Sturdza A et al. Adaptive image guided brachytherapy for cervical cancer: a combined MRI-CTplanning technique with MRI only at first fraction. Radiother Oncol 2013; 107: 75-81.

18. Epstein E, Testa A, Gaurilcikas A et al. Early-stage cervical cancer: tumor delineation by magnetic resonance imaging and ultrasound - a European multicentre trial. Gynecol Oncol 2013; 128: 449-453.

19. Pinkavova I, Fischerava D, Zikan M et al. Transrectal ultrasound and magnetic resonance imaging in the evaluation of tumour size following neoadjuvant chemotherapy for locally advanced cervical cancer. Ultrasound Obstet Gynaecol 2013; 42: 705-712.

20. Testa A, Ludosvisis M, Manfredi R et al. Transvaginal ultrasonography and magnetic resonance imaging for assessment of presence, size and extent of invasive cervical cancer. Ultrasound Obstet Gynecol 2009; 34: 335-344.

21. Schmid MP, Pötter R, Brader P et al. Feasibility of transrecta ultrasonography for assessment of cervical cancer. Strahlenther Onkol 2013; 189: 123-128.

22. Sahinler I, Cepni I, Oksuz DC et al. Tandem application with transvaginal ultrasound guidance. Int J Radiat Oncol Biol Phys 2004; 59: 190-196.

23. Sharma DN, Rath GK, Thulkar $S$ et al. Use of transrectal ultrasound for high dose rate interstitial brachytherapy for patients of carcinoma of uterine cervix. J Gynecol Oncol 2010; 21: 12-17.

24. Stock RG, Chan K, Terk M et al. A new technique performing Syed -Neblett template interstitial implants for gynecologic malignancies using transrectal-ultrasound guidance. Int J Radiat Oncol Biol Phys 1997; 37: 819-825.

25. Mayr NA, Montebello JF, Sorosky JI et al. Brachytherapy management of the retroverted uterus using ultrasound-guided implant applicator placement. Brachytherapy 2005; 4: 24-29.

26. Weitmann HD, Knocke TH, Waldhäusl C et al. Ultrasoundguided interstitial Brachytherapy in the treatment of advanced vaginal recurrences from cervical and endometrial carcinoma. Strahlenther Onkol 2006; 182: 86-95.
27. Granai CO, Allee P, Doherty F et al. Ultrasound used for assessing the in situ position of intrauterine tandems. Gynecol Oncol 1984; 18: 334-338.

28. Davidson MT, Yuen J, D'Souza D et al. Optimization of highdose-rate cervix brachytherapy applicator placement: the benefits of intraoperative ultrasound guidance. Brachytherapy 2008; 7: 248-253.

29. van Dyk S, Garth M, Oates A et al. Reproducibility and interoperator reliability of obtaining images and measurements of the cervix and uterus with brachytherapy treatment applicators in situ using transabdominal ultrasound. Brachytherapy 2016; 15: 71-78.

30. van Dyk S, Kondalsamy-Chennakesavan S, Schneider M et al. Assessing changes to the brachytherapy target for cervical cancer using a single MRI and serial ultrasound. Brachytherapy 2015; 14: 889-897.

31. Narayan K, van Dyk S, Bernshaw D et al. Ultrasound guided conformal brachytherapy of cervix cancer: survival, patterns of failure, and late complications. I Gynecol Oncol 2014; 25: 206-213.

32. van Dyk S, Kondalsamy-Chennakesavan S, Schneider M et al. Comparison of measurements of the uterus and cervix obtained by magnetic resonance and transabdominal ultrasound imaging to identify the brachytherapy target in patients with cervix cancer. Int J Radiat Oncol Biol Phys 2014; 88: 860-865.

33. Van Dyk S, Narayan K, Fisher R et al. Conformal brachytherapy planning for cervical cancer using transabdominal ultrasound. Int J Radiat Oncol Biol Phys 2009; 75: 64-70.

34. Kamrava M. Potential role of ultrasound imaging in interstitial image based cervical cancer brachytherapy. J Contemp Brachytherapy 2014; 6: 223-230.

35. Bernard JP, Metzger U, Rizk E et al. Hysterosonography. Gynecol Obstet Fertil 2002; 30: 882-889 [Article in French].

36. Bongers M, Brölmann, Gupta J et al. Transcervical, intrauterine ultrasound-guided radiofrequency ablation of uterine fibroids with the VizAblate ${ }^{\circledR}$ System: three- and six-month endpoint results from the FAST-EU study. Gynecol Surg 2015; 12: $61-70$.

37. Löhnert M, Doniec JM, Kovács G et al. New method of radiotherapy for anal cancer with three-dimensional tumor reconstruction based on endoanal ultrasound and ultrasound-guided afterloading therapy. Dis Colon Rectum 1998; 41: 169-176.

38. Doniec JM, Schniewind B, Kovács G et al. Multimodal therapy of anal cancer added by new endosonographic-guided brachytherapy. Surg Endosc 2006; 20: 673-678.

39. Christensen AF, Nielsen BM, Engelholm SA. Three-dimensional endoluminal ultrasound-guided interstitial brachytherapy in patients with anal cancer. Acta Radiol 2008; 49: 132-137.

40. Segedin B, Gugic J, Petric P. Uterine perforation - 5-year experience in 3-D image guided gynaecological brachytherapy at Institute of Oncology Ljubljana. Radiol Oncol 2013; 47: 154-160.

41. Fokdal L, Tanderup K, Hokland SB et al. Clinical feasibility of combined intracavitary/interstitial brachytherapy in locally advanced cervical cancer employing MRI with a tan$\mathrm{dem} /$ ring applicator in situ and virtual preplanning of the interstitial component. Radiother Oncol 2013; 107: 63-68. 